\title{
MODELING PROJECTILE MOTION: A SYSTEM DYNAMICS APPROACH
}

\author{
Chand K.K. ${ }^{1}$, Pattnaik S. ${ }^{2}$ \\ ${ }^{1}$ Proof \& Experimental Establishment (PXE), DRDO, Chandipur, Balasore, Orissa, India \\ ${ }^{2}$ Department of Information \& Computer Technology,Fakir Mohan University, Vyasa Vihar, Balasore, Orissa, India \\ E-mail : 'kkchandpxe@indiatimes.com, ${ }^{2}$ pattnaiksabyasachi@yahoo.co.in
}

\begin{abstract}
Modelling projectile motion and its computations has been addressed in many contexts by researchers and scientists in many disciplines in its broad applications and usefulness. It is an important branch of ballistics and involves equations that can be used to work out a trajectory and focuses on the study of the motion bodies projected through external medium i.e. air in space. As system dynamics has been a well-formulated methodology for analyzing the components of a system including causeeffect relationships, therefore the ballisticians interested to apply the system dynamics approach in the armament fields too. In view of above, this paper discusses applications of system dynamics approach for modeling of projectile motion and its simulation from mathematical, physical and computational aspects.
\end{abstract}

Keywords: Artillery, Projectile, Modelling, Trajectory, Simulation, System, and System Dynamics

\section{INTRODUCTION}

In our physical world, motion is ubiquitous and controlled by the application of two principal ideas, force and motion, called dynamics. Through the study of dynamics, we are able to calculate and predict the trajectory of a projectile. This is what science is all about studying the environment around us and predicting the future.

Modelling is the name of the game in physics, and Newton was the first system dynamicist. Modelling is the process of identifying the principal physical dynamic effects to be considered in analyzing a system, through the differential and algebraic equations from the conservation laws and property laws of the relevant discipline [1]. Modelling projectile motion and its simulation is nothing but as a computational tool to calculate the flight path and its characteristics of various projectiles and its families. There is a wide span of trajectory modelling and computation differing widely with respect to their performance and fidelity characteristics, which used throughout the product life cycle of a weapon system to improve the understanding of various aspects of weapon system [2].

The study of projectile modeling and computation is called ballistics. As an applied science, it has a wide meaning and is one of the most exciting and complex fields of science, comprising a wide scope of subjects, ranging from the behaviors of projectile inside a gun barrel (Internal Ballistics), the flight of projectile through the air (External Ballistics) and the interaction between projectile and targets (Terminal Ballistics), shown in Fig .1 Its development is very much related to the evolution of military technology and particularly in artillery projectile system [3][4].

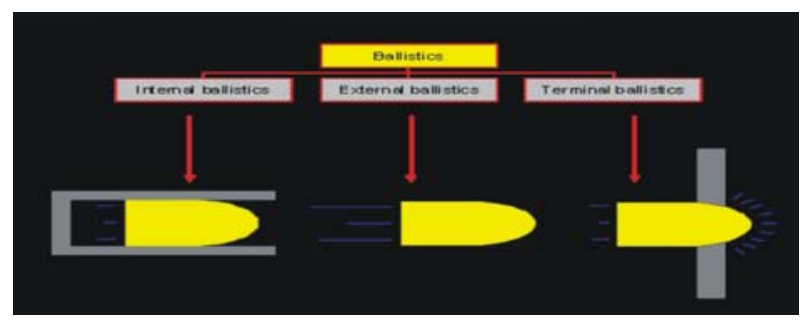

Fig .1 Various Ballistics Environments

The science of investigating projectile trajectory modeling and computation is known as external ballistics or the science of flight dynamics. It existed for centuries as an art before its first beginnings as a science. It is an important branch of ballistics and involves equations that can be used to work out a trajectory and focuses on the study of the motion bodies projected through external medium i.e. air in space [5].

This paper discusses the feasibility of the application of system dynamics approach in the artillery projectile motion analysis under the test and evaluation curriculum activities using a point-mass mathematical model concept by assuming acted upon by gravity and aerodynamic drag, acting in the opposite direction. The model requires only a limited amount of data, namely, one aerodynamic a parameter $\left(\mathrm{C}_{\mathrm{D}}\right)$ as a function of Mach number, the projectile mass and diameter, and simple launch parameters. The resulting equations of motion have been numerically solved to yield the complete trajectory elements of a projectile [6].

In view of above, first the paper explains concept of system and system dynamics. In second the paper discusses importance of system dynamics in artillery projectile motions. Finally, a real time application of system dynamics has been discussed. 
As a matter of policy on the grounds of confidentiality, the model has been normalized and validated with the typical projectile and other data available in published form without loss of generality. One of the authors experience in this area began with the involvement of various trajectory modeling and simulation studies.

\section{PROJECTILE MOTION ENVIRONMENT}

Projectile motion is a fundamental concept, which can be found in introductory physics textbooks. Modelling projectile motion focuses on the details of what a system does. These capture the physical properties of materials, aerodynamics, and simulation of specific systems. They also include interactions between two physical objects or between an object and its environment. A system dynamics approach attempts to understand the physical capabilities of the system at a level that is accurate enough to be used to design the system [7].

Using the state-of-the-art measuring technologies like high-speed camera and computational software, scientists and researchers can investigate the influence of air resistance on for instance the trajectory of a projectile. The motion sequences are digitized and the $x$ and $y$-positions of the projectile in every frame are captured together with the actual time. Comparisons are made with theoretical computations performed for dynamic simulations.

In case of real time phenomenon in armament field, projectile motion is the study of the firing, flight, and effect of ammunition. A fundamental understanding of motion is necessary to comprehend the factors that influence accuracy how to account for them in the determination of firing experimental data. To ensure accurate predicted fire, we must strive to account for and minimize those factors that cause round-to-round variations, particularly, muzzle velocity. Fig .2 shows an exterior ballistics environment of a projectile system. Similarly, Fig .3 depicts the various motion concepts in space [6][7].

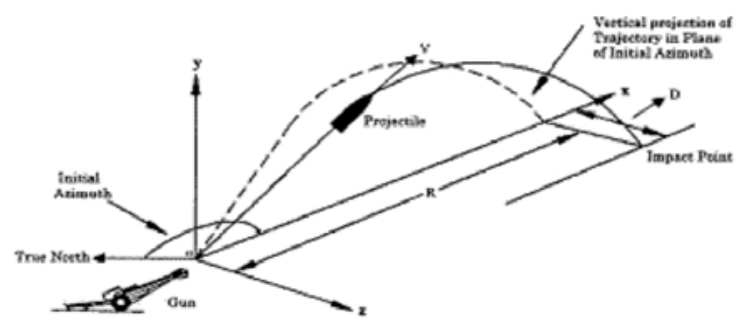

Fig . 2 Projectile Motion in External Environment

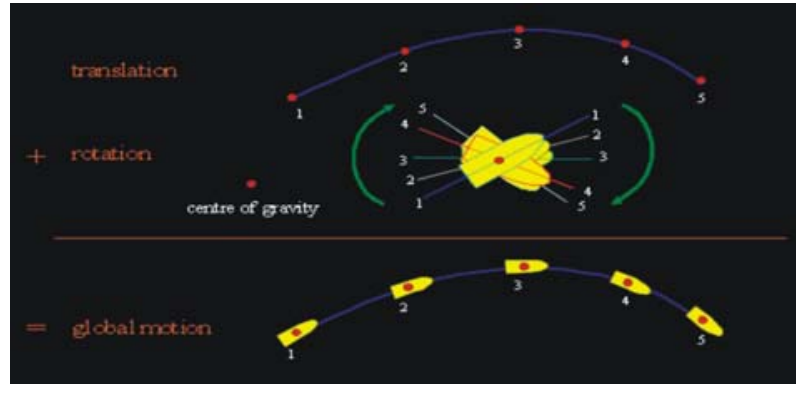

Fig .3: Translation and Rotation Motions of a Projectile System

\section{CONCEPTS OF SYSTEM AND DYNAMICS}

We live in a world of systems driven by cause and affect, which include physical, biological, social and so on. Man always searches for unfolding the truth of nature and for capitalizing on natural endowments for his own knowledge and use by following the principle of reduction, analysis, and summation over for long period. Systems can be modeled as nodes representing system variables and connecting lines representing causal effects. The changing value of one variable can cause another to increase or decrease as described by equations. Understanding how a system really works is the first step towards using, improving, automating or explaining it to others [8][9].

Learning the system dynamics approach requires that researchers, scientists or modelers understand the concept of a system and the terms of dynamics. The term 'system' is a very broad and general concept that relates to various areas such as social systems, technological systems, and natural systems. Therefore, this subject has been studied from different angles and points of interests. A system is an entity that maintains its existence and functions as whole through the interaction of its parts[10].

\section{A. Definition}

The Greek origin of the word 'system' is systema, which is derived from syn meaning together and histema meaning 'to set'. Thus system literally means 'to set together'. It is simple a collection of interacting elements for which there are cause and effect relationships among the variables. A well-understood definition of a system is a "set of elements joined together for a specific purpose." More elaborately, a system is defined as "a group of interacting, interrelated, or interdependent elements forming a complex whole to perform a certain objective." The term "system" is being used in different fields in a variety of meanings [11][12].

\section{B. General Characteristics of Systems}

The general characteristics of systems can be stated as [12][13][14]: 
(a) Systems consist of (definable) elements;

(b) Between these elements there exit (mostly functional) interrelations;

(c) Every system has a boundary to the surrounding "environment", which is more or less permeable, material or immaterial.

(d) Systems often have a dynamic behavior over time. This behavior is often related to the aim of the system.

(e) System elements might be considered as whole sub-systems or a system might be a single element of a larger system. Thus whole hierarchies of systems may emerge.

\section{Classification}

Systems generally can be classified as[15]:

(a) Static Systems have an output response to an input that does not change with time.

(b) Dynamic Systems have a response to an input that is not instantaneously proportional to the input or disturbance and that may continue after the input is held constant. Dynamic systems can respond to input signals, disturbance signals, or initial conditions. The Figure 4 shows a general concept of a dynamical system.

\section{Categories}

Systems can be usefully categorized into three general classes as: (a) Lumped Systems, (b) Distributed Systems, (c) Discrete Systems. These can be differentiated based on their inputs and outputs, state variables, their elements, and the mathematics useful for their analysis [11][15].

\section{SYSTEM DYNAMICS}

System Dynamics (SD), initially named Industrial Dynamics, a computer-based simulation modelling methodologies, is defined as 'the study of the informationfeedback characteristics of industrial activity to show how organizational structure, amplification (in policies), and time delays (in decisions and actions) interact to influence the success of the enterprise' [12] [16].

System dynamics deal with:

the mathematical modeling of dynamic systems; and

- response analyses of such systems with a view toward understanding the dynamic nature of each system and improving the system's performance;

System dynamics is an academic discipline created in the 1960s by Forrester of the Massachusetts Institute of Technology. It is fundamentally interdisciplinary and grounded in the theory of nonlinear dynamics and feedback control developed in mathematics, physics and engineering.

Using system dynamics approach the researchers and scientists produce simulation tools called as micro worlds to make certain experiments by replacing for the real world. That is why they are called as micro worlds. The experiments in the micro worlds can be repeated easily using varying parameters and alternative scenarios, which allows the researchers to see how the dynamics of the system works, by experiencing it in the virtual world. The experiments are done with the help of certain easy to use simulation software. STELLA is a computer simulation program, which provides a framework and an easy- to-understand graphical interface for observing the quantitative interaction of variables within a system. The graphical interface can be used to describe and analyze very complex physical, biological, and social systems [14].

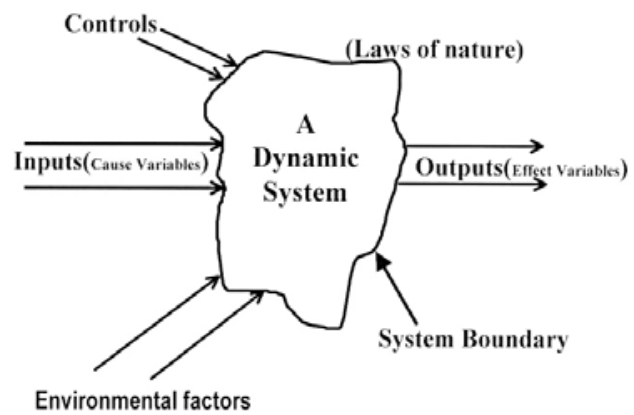

Fig .4 A Dynamical System

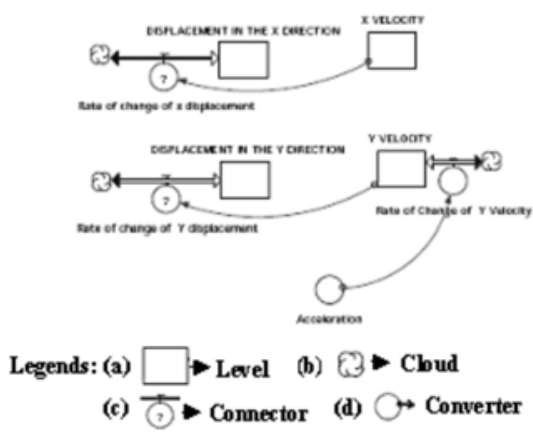

Fig .5 The System Dynamic Language 
The language of system dynamics is simple, which is shown in Fig .5 Levels (also called stocks) contain quantities describing the state of the system. If the model (or system) were stopped, each of these would continue to hold its quantity for observation. For example, the kinematics model in motion system. In a typical system dynamics diagram, these appear as labeled rectangles. If the value of a particular state (stock/initial values) is of no concern to the problem at any instant, then the state (stock) is shown as a cloud to indicate that it's outside the boundary of the model.

Rates (also called flows) are the inflows to and outflows from the various levels. These appear as labeled valves on pipes connecting levels. If the system were stopped, rates also stop. For example, the velocity ( $x$ or $y$ direction) is a rate of change of displacement ( $x$ or $y$ direction). If the system were frozen in time, there would be no flow of motion at all.

Connectors and converters (also called auxiliaries) measure the quantities in levels and, through various calculations, control the rates. These appear as lines with arrows and as circles. Projectile motions are modeled by these calculations. Let us build two simple models in the kinematics by defining the rate of change of each variable. Let us create two new stocks for the velocities in the $x$ and $y$ directions. We know that the velocity in the $x$ direction is a constant, however, the velocity in the $y$ direction varies over time due to the acceleration due to gravity. The model looks like as follows [10]:

\section{A. System Dynamics in Projectile Motion}

System dynamics is a general and user-friendly approach to the modeling of dynamical systems, irrespective of the field of applications. Although system dynamics modelling has been practiced and applied in lots of fields outside areas- is hardly known in external ballistics. Having inspired by successful policy changes in lots of fields, the system dynamics researchers targeted to apply the system dynamics approach in the ballistics fields too. External Ballistics particularly in projectile motion is suited to the methodologies and tools of system dynamics and system thinking. There is a remarkable increase in the interest and understanding level of the scientists and researches using system dynamics approach. It is a wellformulated methodology for analyzing the components of a projectile system including cause-effect relationships and their underlying mathematics and logic, time delays, and feedback loops. Elements of systems interact through feedback loops as the buildings blocks of all dynamic systems, which consist of accumulation, or level variables, rate variables that change levels, and information connecting levels to rates. Moreover the researchers and scientists understand that there are usually more than one cause and effect relationships under the complex systems. In addition, researchers and scientists see that the result of the combined interactions of all the cause and effects relationship cannot be analyzed by superficial studies [15][17].

\section{B. Projectile as a Dynamical System}

A projectile is defined as "an object, projected by an applied exterior force or impulse and continuing in motion by virtue of its own inertia over a very short period of time". A projectile with its weapon and ammunition form a projectile system. It is term that emerges from many disciplines and domains and has many interpretations, implications and problems associated with it. Generally, modern artillery projectiles have many features in common, which include a fuze, ogive, bourrelet, and rotating band shown in Figure 6. Similarly, Figure 7 shows the concept of a dynamical projectile system.

A trajectory is an imagined trace of positions followed by an object moving through space. A projectile system, when subjected to propelled, fired, shot, launched, or thrown through space by the exertions of a force by a suitable means, behaves dynamically. This is a dynamic system, because the level of the elements changes in time. Mathematically the term trajectory refers to the ordered set of states (as a function of the time), which are assumed by a dynamical system over time. The trajectory of a projectile depends upon many factors, such as the components of initial velocity of the projectile, its weight, shape, size, rate of spin, the density and temperature of the air, the direction and velocity of the wind at various altitudes, and the latitude of the gun and the azimuth of fire. Most of these factors are capable of taking many values. Mathematically a trajectory may be described either by the geometry of the path (i.e. the set of all positions taken by the object), or as the position of the object over time

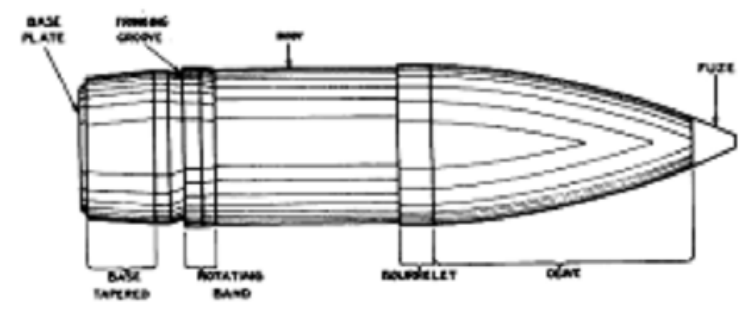

Fig .6 Different sections of a projectile 


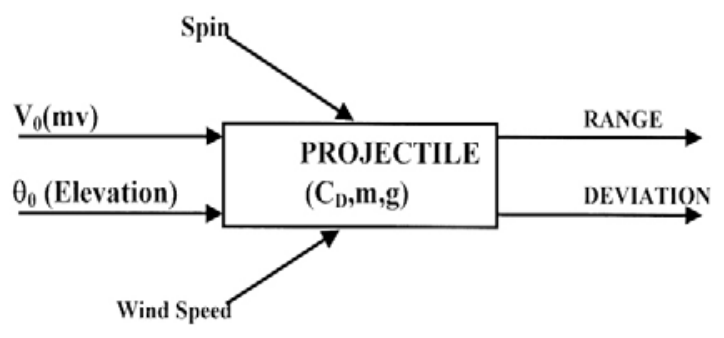

Fig .7 Excitation and response of a projectile System

To describe a trajectory completely it is sufficient to specify the $x, y$ and $z$ coordinates of the center of gravity of the projectile at any time $t$ after the release by the projecting mechanism; in other words, a trajectory is regarded as defined by: $x=x(t) ; y=y(t) ; z=z(t)$; where $x(t)$, $y(t)$ and $z(t)$ are functions of the time $t$ which are equal to zero when $t=0$.

The trajectory is determined by [4]:

(a) the position of the origin;

(b) the conditions of projections;

(c) the ballistic characteristics of the projectile;

(d) the characteristics of the air through which it passes;

\section{Projectile Trajectory Modelling and Simulation}

Trajectory simulation, in the present context, means computing the flight path and other parameters, such as range, deviation, orientation, and angular rates of a projectile from the start to the end of its motion. Trajectory simulation deals with mathematical models of the behavior of projectile and its subsystems (if any) during its test \& evaluation and operations. The equations of motion determine the acceleration, velocity and position (range \& deviation) of the projectile resulting from forces and moments due to gravity, thrust and aerodynamics. The guidance and control models account for subsystems such as the control system in case of a missile system.

Particularly trajectory simulation requires a customized approach. The level of sophistication of simulations varies greatly depending on the applications ranging from unsophisticated two-dimensional models to very detailed six-seven degree of freedom models as per shapes and sizes of projectile systems. The requirements of a given trajectory simulation are derived from the objectives of the intended users requirements like, analysis, development, procurement and operation[18][19].

\section{Mathematical Modelling}

On account of the complicated nature of the resistance of the air, an accurate mathematical prediction of the trajectory is difficult. Some of the difficulties arise from the fact that the projectile is of finite size. To avoid these, we regard the projectile as a particle. A mathematical model with simplified point mass/simple particle trajectory model is proposed to simulate a plane projectile trajectory by considering following basic assumptions [4][5][6]:

2iy The earth is assumed to be flat i.e., the curvature influence of the earth is ignored;

297 The projectile is planer motion;

2iy The wind speed is assumed to be zero;

2iy The drag index and mass of the projectile are all constants during firing;

2iy The drag force is proportional to the square of the instant velocity;

2iy The drag coefficient and density of air are assumed to be constant during firing;

2y The force of gravity is independent of the altitude, i.e. the gravity force is constant;

29y The forces, like centrifugal, coriolis, magnus forces and its cross-effects are assumed to negligible;

By applying the systematic system dynamics steps [16], the motion of a projectile of mass $m$ influenced by gravity $g$ and air resistance js described by

$a_{x}=\frac{d^{-} x}{d t^{2}}=-\frac{1}{m} \cdot F_{D} \cdot \cos \theta$

$a_{x}=\frac{d^{2} y}{d t^{2}}=-\frac{1}{m} \cdot\left(F_{D} \cdot \sin \theta+m \cdot g\right)$

Where, $v=\sqrt{v_{x}^{2}+v_{y}^{2}} u=\tan ^{-1}\left(\frac{v_{y}}{v_{x}}\right)$ and $F_{D}=\frac{1}{2} \cdot \rho \cdot v^{2} \cdot A \cdot C_{D}$

The above equations are solved subject to the following state or initial conditions:

$u=\frac{d x}{d t}=v_{0} \cos \theta_{0} ; v=\frac{d y}{d t}=v_{0} \sin \theta_{0}$

The drag force $F_{D}$ acting on the projectile depends on air density $\rho$, velocity $v$, cross-section area $A=\pi \times r^{2}$, radius of the projectile $r$, and drag coefficient $C_{D}$. If the drag force is neglected, the calculation of the projectile trajectory becomes trivial. If however the drag force is taken into account, the analytical solution is not soluble due to the 
drag dependency on the square of the velocity and presence of angle as argument of trigonometric functions. With the aid of computers it is possible to solve the equations numerically, either by using a generalpurpose program such as Microsoft Excel or $\mathrm{C} / \mathrm{C}++$ or dedicated programs for dynamic simulations, e.g. MATLAB/SIMULINK or Powersim or STELLA to generate the trajectory elements [20][21].

\section{E. Simulation Modelling in Projectile Motion}

In projectile modelling and simulation, the influence parameters such as muzzle velocity, angle of inclination, drag coefficient, atmospheric conditions and projectile shape \& size have been considered for estimation of trajectory elements. The governing plane motion equations of the mass center of the projectile are written using Newton's Second Law of motion ( $F=\mathrm{ma}$ ) and then the numerical integration is carried out using fourth order Runge-Kutta (RK) method by assuming the initial values of the speed $v$ and the angles are known in Equation 2. Finally, the knowledge of accelerations is used to calculate changes in velocity components over a shorttime step and these in turn are used to calculate changes in co-ordinates in the $x$ \& y directions using Equation 1 and appropriate graphs are plotted. Results of these simulation results are compared with measured values through Doppler Radars and high-speed camera instruments. The two components of motion are split into a vertical (left) and a horizontal (right) part. Fig .8 shows the simulation model used to simulate the motion of a projectile. Fig .9, Fig .10 and Fig .11 show the how the drag coefficient varies with speed and computed trajectories together with a graph depicting the speed of the projectile

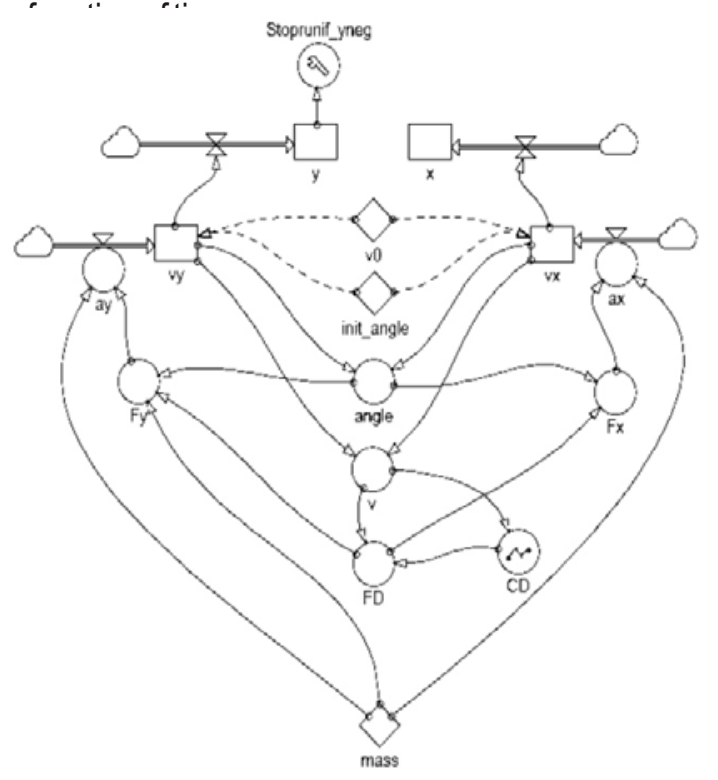

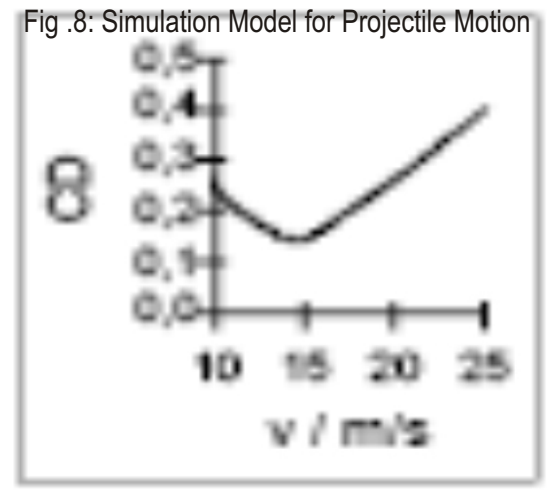
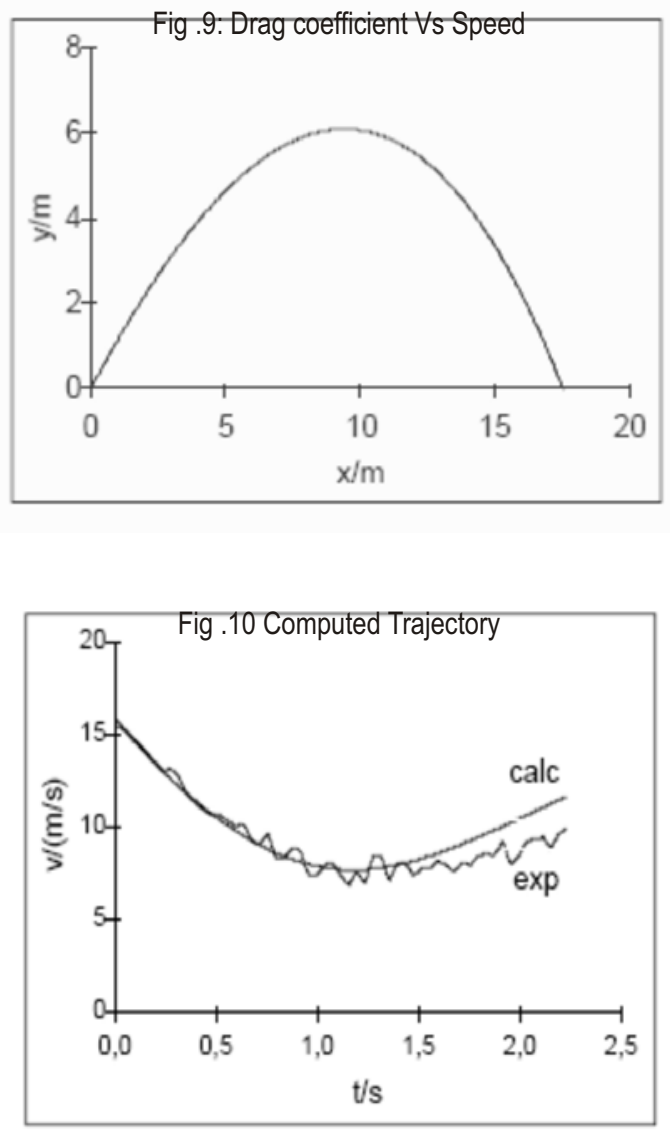

Fig .11: Computed Vs Experimental Speed of the Trajectory

\section{CONCLUSION}

System dynamics is a general approach for defining and solving problems. This approach consists of two parts: building the model and testing it. Both parts employ mathematical equations. The relationships between the elements are established through the mathematical relations. The present study has been carried out to simulate the trajectory elements of a projectile system using point mass concept by considering drag force and 
projectile mass as main forces acting on the projectile. The model has been simulated for different initial velocities and angle of elevations which shown in the graphs. System dynamics, a very big idea, is not quick and easy to learn. It takes time, patience, good instruction and follow-up support. The system dynamics technique proved to be an efficient approach for the simulation of a projectile system. Its merits include the increased speed of model development, ease of model improvement, the ability to simulate the interactions between the model components. The efficiency of the developed method gives satisfactory results compared with published data of verified experiments and computational codes on system dynamics model analysis of various trajectories of artillery projectile systems.

\section{REFERENCES}

[1] RL Woods and KL Lawrence, 1997, Modelling and Simulation of Dynamic Systems, Prentice Hall, Inc, New Jersey.

[2] Driels, M. R., 2004, Weaponeering: Conventional Weapon System Effectiveness, $1^{\text {st }}$ Edition, AIAASeries, Inc, Virginia.

[3] Fundamental of Ballistics-Gunther Dyckmans (http://users.telenet.be/gd.ballistic.htm).

[4] G.M.Moss,D.W.Leeming and C.L.Farrar, 1995, Military Ballistics: A Basic Manual-, Brassy's Ltd series, Vol-I, UK, London.

[5] 1987, Text of Ballistics and Gunnery-I, Her Majesty's Stationary Office, London.

[6] Chand K.K., Panda H.S., 2007. "Mathematical Model to Simulate the Trajectory Elements of an Artillery Projectile Proof Shot", Defence Science Journal , 57, No1, January, 139-148.

[7] U Durak, H Oguztuzun and SK Ider, 2006, An Ontology For Trajectory SimulationProceedings of the Winter Simulation Conference, pp-1160-1167.

[8] The Potential of System Dynamics -ANew Era of Strategic Planning, Leading Edge, Issue 10, November 2005.

[9] DV Mehta, 2007, System Evolution: Concepts \& Applications -, CSI, Communication, Volume No-31,Issue No-7, October, pp-26-28.

[10] G.W. Winch, 2000, System Dynamics: from Theory to Practice $-1^{\text {st }}$ International Conference on Systems Thinking in Management.

[11] PKJ Mohapatra, P Mandal and MC Bora, 1994,
Introduction To System Dynamics Modelling -, University Press (India) Limited.

[12] Forrester, J.W., 1961, Industrial dynamics, MIT Press, Cambridge, MA, USA.

[13] PE Wellstead, 2000, Introduction to Physical System Modelling, Academic Press Ltd, London.

[14] System Dynamics Approach in Science and Technology Education- Hasret Nuhoğlu, Mert Nuhoğlu, Journal of Turkish Science Education, Volume 4, Issue 2, September 2007.

[15] DW Boyd, 2001,System Analysis and Modelling -Academic Press, San Diego, CA 92101- 4495, USA.

[16] Wolstenholme, E.F. and R.G. Coyle, 1983, The Development of System Dynamics as a Methodology for System Description and Qualitative Analysis. Journal of the Operational Research Society, 34(7): p. 569.

[17] N Hritonenko and Y Yatsenko, 2003, Applied Mathematical Modelling of Engineering Problems-KluwerAcdemic Publishers, USA.

[18] Cloud and Rainey, 1998, Applied Modelling and Simulation: An Integrated Approach to Development and Operation, The McGraw-Hill Companies, Inc.

[19] A Report, 2000, Guidelines: Use of Modelling and Simulation to Support Test and Evaluation, U.S. Army, Washington, D.C.

[20] Harold Klee, 2007, Simulation of Dynamics Systems with MATLAB and SIMULINK-,CRC Press, New York.

[21] M Nuruzzaman,2004, Modelling and Simulation in SIMULINK for Engineers and Scientists- Author House, Indiana. 